https://doi.org/10.15407/ujpe65.9.784

O.P. ARTYKULNYi, ${ }^{1,2}$ V.I. PETRENKO ${ }^{3}$ M.V. AVDEEV ${ }^{2}$ L.A. BULAVIN ${ }^{1}$

1 Taras Shevchenko National University of Kyiv, Faculty of Physics

(4, Academician Glushkov Ave., Kyiv 03022, Ukraine; e-mail: artykulnyi@gmail.com)

2 Joint Institute for Nuclear Research

(6, Joliot-Curie Str., Dubna 141980, Russia)

${ }^{3}$ BCMaterials, Basque Centre for Materials, Applications and Nanostructures

(UPV/EHU Science Park, Leioa, Spain)

\title{
COMPLEXES OF SURFACTANT MICELLES WITH POLYMERS IN AQUEOUS LIQUID SYSTEMS
}

\begin{abstract}
Researches on the structural self-organization and aggregation in aqueous liquid systems of surfactants and polymers have been reviewed. Main attention is focused on the results obtained for the interaction of ionic surfactants with non-ionic polymers. Capabilities of the small-angle neutron scattering and neutron reflectometry methods for the study of the micellar structure, micelle-polymer aggregates, and complex multicomponent systems with nanoparticles are considered. Brief information is provided on some complementary methods used for the structural analysis of liquid systems with self-organization.

Ke ywords: micelles, surfactants, surfactant-polymer complexes.
\end{abstract}

\section{Introduction}

Liquid systems of surfactant micelles are a matter of concern in modern researches carried out in molecular physics, physical chemistry, biophysics, and some other academic disciplines. Considerable attention to systems with self-organization that are based on the solutions of surfactants and polymers is associated, first of all, with unique properties of nano- and micro-sized micelles, which emerge as a result of the intermolecular interaction, and whose structure and properties are quite sensitive to variations of the thermodynamic system parameters $[1,2]$. Such systems find wide application in various industrial sectors, e.g., oil production, cosmetics, pharmaceutics, and food technology.

The spontaneous formation of micelles in surfactant solutions with a critical micelle formation (micellization) concentration (CMC) and the formation of surfactant-polymer aggregates in solutions with a critical micelle aggregation concentration (CAC) are described in terms of the nucleation of a new microphase of hydrophobic domains (the mass action law) [1]. In so doing, micelles are considered as dynamic structures with an average half-life of $10^{-3} \mathrm{~s}$.

(C) O.P. ARTYKULNYI, V.I. PETRENKO, M.V. AVDEEV, L.A. BULAVIN, 2020
A thermodynamically equilibrium state of every micellar system is characterized by such parameters as the average aggregation number $N_{\text {agg }}$, i.e. the average number of monomers entering the structure of one micelle, and the ionization degree $\alpha$, which is equal to the fraction of dissociated molecules of an ionic surfactant in the micelle composition. In micellar systems with surfactant concentrations close to the CMC, micelles have a spherical shape with a radius equal to the length of the hydrophobic part of the surfactant molecule. In the case of saturated hydrocarbons, this length can be estimated with the help of the semiempirical Tanford formula [3].

As the surfactant concentration in the system increases, or a salt is added. or the solution $\mathrm{pH}$ changes, micelles considerably grow in size, and their morphology changes (Fig. 1, a). There arises ellipsoidal, cylindrical, and branched micelles, vesicles, lamellae, and so forth [4]. A tendency for a micelle to grow and acquire a particular shape is described to a great extent by the critical packing parameter (CPP). Its geometrical sense corresponds to the ratio between the monomer volume $v$ and the product of the molecular length $l$ by the area $a$ of the polar part crosssection. In non-polar solvents, the formation of socalled reverse micelles is possible. They consist of an external non-polar shell and polar groups concentrated in the micelle core (inside the micelle).

ISSN 2071-0194. Ukr. J. Phys. 2020. Vol. 65, No. 9 

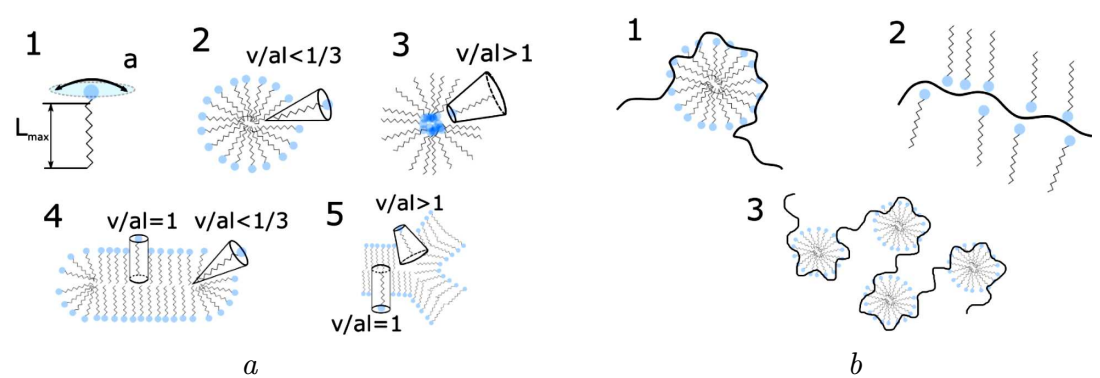

Fig. 1. Schematic representation of micellar structures $(a)$ : surfactant molecule (1), spherical micelle (2), reverse micelle (3), cylindric micelle (4), and branched micelle (5). Schematic representation of surfactant-polymer complexes $(b)$ : with surfactant micelles (1), with surfactant monomers (2), and a structure with several micelles (3)

Despite that the fundamentals of modern ideas concerning the surfactant micellization in liquid systems with neutral polymers [5] and polyelectrolytes [6, 7] were elaborated as long ago as in the 1970-1980s, they still remain relevant and provide a solid basis for the study of multicomponent systems [8,9]. Depending on the surfactant type (nonionic, cationic, anionic, zwitterionic) and the type of a polymer interacting with surfactant molecules (nonionic, polyelectrolyte), various adsorption mechanisms can take place. They are described by three typical binding isotherms: cooperative, non-cooperative, and anti-cooperative [10]. In all cases, surfactant molecules can be incorporated into the complex structure both alone or as micelles (Fig. $1 b)$.

\section{Experimental Methods for Studying Surfactants and Surfactant-Polymer Complexes}

Since the process of micelle and surfactant-polymer complex formation simultaneously affects various physicochemical properties of the system, it can be studied making use of a number of experimental methods [11]. This approach makes it possible to confront, compare, and complement the results obtained in the framework of different methods. Phillips [12] proposed a unified approach for determining the CMC value by analyzing various experimentally observed quantities $\varphi$ as unctions of the surfactant concentration $C$. Namely, he defined the $\mathrm{CMC}$ as a concentration at which the slope of the dependence $\varphi(C)$ changes most rapidly:

$$
\left(\frac{d^{3} \varphi}{d C^{3}}\right)_{C=\mathrm{CMC}}=0 .
$$

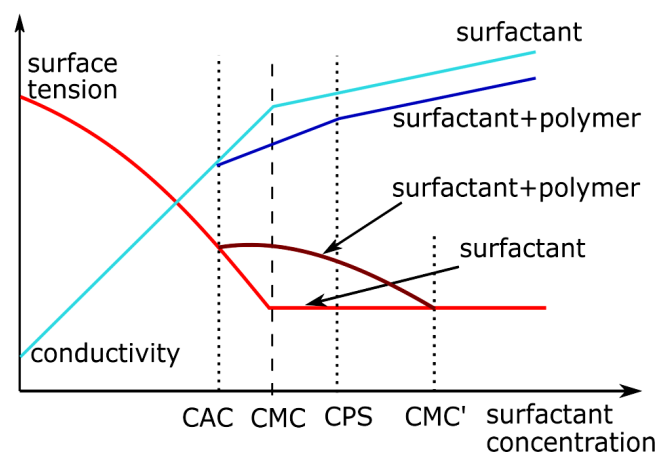

Fig. 2. Schematic representation of typical dependences of the solution surface tension and conductivity on the surfactant concentration and how the addition of a neutral polymer affects them. $\mathrm{CAC}$ is the critical aggregation concentration, CMC the critical micelle concentration, CPS the concentration of polymer saturation, $\mathrm{CMC}^{\prime}$ the shifted $\mathrm{CMC}$ in the surfactantpolymer system

Such methods as surface tension measurements, conductometry, and fluorescence spectroscopy are used most extensively, when studying the surfactant and surfactant-polymer systems. A schematic representation of the experimental dependences of the conductivity and surface tension on the surfactant concentration in micellar liquid systems with and without adding a neutral polymer is depicted in Fig. 2 .

The surface tension of surfactant solutions is unambiguously determined by the concentration of surfactant monomers which are actively adsorbed at the liquid/air interface and, in such a way, minimize the surface energy. At solution concentrations higher than the CMC, the addition of surfactant into the solution does not change the surface tension of the latter, because all excess molecules become involved into the micellization. Assuming that the surfactant solution 


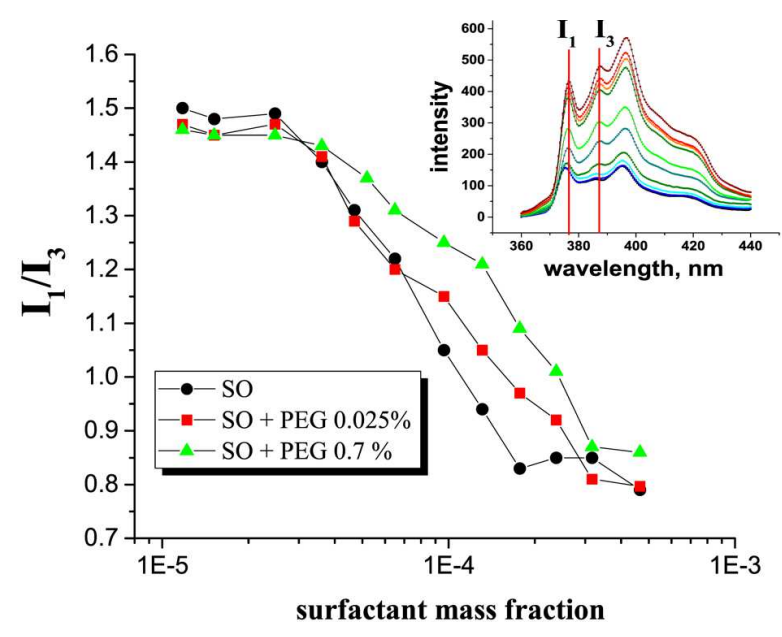

Fig. 3. Experimental dependences of the polarity parameter on the sodium oleate (SO) concentration determined from pyrene fluorescence spectra (inset) in the presence of the neutral PEG polymer with a molecular weight of $35 \mathrm{kDa}$ with various concentrations

is ideal, and the adsorption process is described by the Langmuir isotherm, we can obtain the following expression for the surface tension $\sigma$ as a function of the surfactant monomer concentration in the solution, $x_{i}$ :

$\sigma=\sigma_{0}-R T \Gamma_{\infty} \log \left(1+K_{s} x_{i}\right)$,

where $\sigma_{0}$ is the surface tension of the solvent, $R$ the universal gas constant, $T$ the absolute temperature, and $\Gamma_{\infty}$ the surface excess of surfactant molecules at the saturation.

If a polymer is added to a liquid surfactant solution, the application of Eq. (1) requires that the dependence of the parameter $x_{i}$ on the total surfactant concentration in the system, $X_{t}$, and the polymer concentration, $P_{t}$ - i.e. $x_{i}=x_{i}\left(X_{t}, P_{t}\right)-$ has to be taken into consideration, because some of the surfactant monomers interact with the polymer and create complexes. Theoretical models describing the interaction between the surfactant and the polymer, and determining the balance of surfactant monomers make it possible to obtain the dependence $\sigma=\sigma\left(X_{t}, P_{t}\right)$, which can be measured experimentally [13].

Molecules of ionic surfactants dissociate well in water, and their solutions have the properties of classical electrolytes. Therefore, it is possible to determine the characteristic $\mathrm{CMC}$ and $\mathrm{CAC}$ parameters for ionic surfactants by measuring the specific conductance $\kappa$. This parameter has a linear dependence on the concentration, $\kappa=\kappa(C)$, the slope of which changes at the CMC [14]. In systems with the addition of polymer, the curve slope changes twice: at the $\mathrm{CAC}$ and at the concentration of polymer saturation (CPS) [15].

The fluorescence spectroscopy method [16], when being applied to study the surfactant micellization, is based on the measurement of the radiation spectrum emitted by a probe substance that was added to the system and whose molecules are sensitive to the polarity of the microenvironment. Such an aromatic dye as pyrene is widely used as a probe, because it is well soluble in hydrophobic domains of heterogeneous systems, and the ultraviolet radiation absorbed at $338 \mathrm{~nm}$ invokes its fluorescence spectrum consisting of five broad bands. The first (at $372 \mathrm{~nm}$ ) and the third (at $384 \mathrm{~nm}$ ) of those bands are sensitive to the dielectric permittivity constant of the microenvironment. Their intensity ratio $I_{1} / I_{3}$ is called the polarity parameter and can be directly measured in the experiment (Fig. 3). In a polar environment, the polarity parameter acquires values within an interval from 1.5 to 2 , being smaller than unity in a non-polar environment. A monotonous decrease of the polarity parameter with increasing the surfactant concentration may testify to the formation of micelles and the transition of pyrene molecules from the solvent to the hydrophobic micelle core.

\section{Structural Studies of Multicomponent Liquid Systems with Surfactants}

Magnetic liquid systems are suspensions of magnetic particles coated with a stabilizing layer of anionic surfactant molecules such as sodium oleate, lauric acid, or dodecylbenzenesulfonic acid [17-19]. They have medical applications, in particular, in the hyperthermal tumor removal [20] and tomographic imaging [21]. To improve the biocompatibility of magnetic liquid systems, the surface of nanoparticles is covered with a polymer layer of polyethylene glycol (PEG) [22]. The latter has protein-resisting properties and counteracts the rapid removal of magnetic particles from the organism [23]. The type of surfactant molecules and the parameters of the corresponding micellar system substantially affect the stability of the magnetic liquid system [24]. Addition of polyethylene glycol to magnetic fluid systems changes the structure of aggregates of magnetic particles in the 
system bulk [25] and violates the surfactant monomer balance, which results in the stability reduction of the whole system.

It should be noted that only a definite concentration ratio between the added polymer and the magnetic nanoparticles (amount of the used surfactant) invokes a structural reorganization in the aqueous ferrofluids of this type. With the help of neutron reflectometry, a considerable difference was demonstrated between the structural organization of particles at the ferrofluid/solid interface in initial aqueous magnetic fluid systems (with only surfactant molecules in the system) and after their modification with the PEG polymer [26]. It should also be noted that, according to neutron reflectometry data, the initial aqueous magnetic liquid system and the highly stable ferrofluid on the basis of non-polar solvent have similar behavior at the ferrofluid/solid interface [27]. Only the adsorption of separate nanoparticles rather than their aggregates was observed in such systems.

The influence of a surfactant on the structural organization of aqueous ferrofluids was analyzed on the basis of the small-angle neutron scattering (SANS) method. Aggregates of various sizes and types were observed for magnetite magnetic nanoparticles in deuterated water, if the system was stabilized with sodium oleate or dodecylbenzenesulfonic acid [24]. The surface tension measurements made it possible to detect a substantial difference between the critical micellization concentrations for the surfactants of two types. The difference between the aggregate structures revealed in ferrofluids was associated with the presence of surfactant molecules in the aqueous solutions. In particular, this difference concerned the parameters of micelle structure and interaction, such as the aggregation number, the micelle charge and average size, the surface potential, and others [28, 29].

Despite a wide range of solvents that are used as a liquid basis for ferrofluids, the controlled synthesis of highly stable aqueous magnetic liquid systems under conditions close to neutral ones still remains a problem. A straightforward way to impede the aggregation consists in the application of the so-called steric repulsion arising when magnetic nanoparticles are coated with one or more surfactant layers. As a result, the attraction forces in the system diminish owing to the growth of the average distance between the magnetic particles, which prevents the aggregation in ferrofluids. Note that the structure and aggre- gation stability of the magnetic fluid system usually depend on the type of a surfactant and its content in the system.

As was shown earlier [30,31], the stabilization efficiency and the size distribution of magnetic particles in non-polar ferrofluids depend on the surfactant type. A correlation between the efficiency of the surfactant as a stabilizer and the structure of non-polar magnetic liquid systems with an excess of a surfactant was detected with the help of SANS [30,32].

For polar ferrofluids, including aqueous magnetic liquid systems, a certain excess of a surfactant is required in order to create the second layer of surfactant molecules on the surface of magnetic nanoparticles and provide the so-called double-layer steric stabilization. In this case, as was shown in previous SANS studies of the efficiency of various surfactants for the stabilization of aqueous ferrofluids [33], the type of a surfactant and its amount also affect the structure and stability of the magnetic liquid system.

The SANS method was used to detect the scattering signal from micelles (sodium oleate and dodecylbenzenesulfonic acid) in the bulk of the aqueous magnetic liquid system. The volume fraction of a surfactant in micelles, $\varphi_{\text {mic }}$, was estimated following the procedure proposed in work [28] for the structural analysis of the micellization in technical aqueous ferrofluids with the double-layer stabilization by means of dodecylbenzenesulfonic acid.

We emphasize the basic importance of SANS applications in the discussed researches of multicomponent systems with surfactants. The SANS results obtained for aggregate sizes are similar to the results given by the electron microscopy and dynamic light scattering methods. The application of the contrast variation in the neutron method made it possible to understand the internal structure of aggregates. In the absence of SANS data and according to the results of the microscopy or dynamic light scattering method, the aggregate size growth after the introduction of PEG into a magnetic liquid system could be interpreted as the formation of a PEG shell around the magnetite particle, with the shell thickness being an order of the polymer molecule length. However, as follows from the SANS data, this rather popular interpretation of light scattering data is invalid in essence, so that the hydrodynamic size determined from light scattering data should be considered as characterizing the aggregation stability of a colloidal system. 
By an example of silicon nanoparticles, protein, and surfactants, it was shown for three-component systems that the synergistic character of the interaction between the system components - despite that all components have the same electrical charge sign - allows the formation and coexistence of complex structures such as nanoparticle aggregates together with surfactant-polymer complexes to take place [9]. A capability to form complexes of the polyvinylpyrrolidone (PVP) polymer with the anionic surfactant sodium dodecyl sulfate (SDS) makes it possible to control the properties of the polymer layer adsorbed on the surface of silicon nanoparticles, whereas the degree of interaction between the anionic surfactant and the neutral polymer can be varied by adding a non-ionic surfactant (alcohol ethoxylate, C13E7) that forms micelles, mixed with SDS, and reduces their electrostatic interaction [8]. Surfactant-polymer complexes also play an important role in drug delivery systems [34], where they can improve the solubility of pharmacological components and prevent their precipitation [35].

In the case of semidilute polymer solutions, i.e. at concentrations of an order of the polymer chain overlap concentration $C^{*}$, and under certain conditions, micelles of an ionic surfactant can play the role of binding links between separate polymer molecules, thus effectively forming a polymer network with rheological properties similar to those of gel [36]. The process of viscoelastic gel formation depends on the solution temperature, and it is reversible [37]. The research of the viscosity of such solutions revealed a substantial increase of their shear viscosity, which is associated with the network appearance. This is unlike the case of dilute polymer solutions $\left(C<C^{*}\right)$, where the process of micelle aggregation results in a reduction of the solution viscosity [38].

In work [39] dealing with the study of the aggregation number $N_{\text {agg }}$ of SDS micelles in the presence of the neutral polyethylene oxide (PEO) polymer with a molecular weight of $20 \mathrm{kDa}$, it was shown making use of the fluorescence spectroscopy method that the interaction of micelles with the polymer leads to a significant (several times) decrease of $N_{\text {agg }}$ and an increase of the polarity parameter $I_{1} / I_{3}$. Those variations testify that the micelle aggregates become smaller in size, and the aggregation is performed by a sparse hydrophobic domain into which the solvent molecules can better penetrate. However, a similar reduction of the micelle size also takes place in solutions with the addition of monomers. The latter was shown using the fluorescence spectroscopy and SANS methods by an example of acrylamide monomers and SDS surfactant [40], where acrylamide molecules are built into the membrane shell and increase its ionization degree.

An important characteristic of surfactant polymer systems is the molecular weight of a polymer. In work [41], using the classic SDS + PEO system as an example, the dependences for the binding isotherms were obtained, and it was shown that the aggregation takes place, if the polymer molecular weight exceeds $1 \mathrm{kDa}$. At higher molecular weights, the CAC value gradually decreases and reaches a constant value at a molecular weight of $8 \mathrm{kDa}$. Besides the polymer chain length, the ability of a polymer to form complexes also strongly depends on the stiffness parameter or the polymer persistent length, which affects the maximum possible number of surfactant molecules aggregated at one polymer molecule and the optimal aggregation number of the micelle itself in the formed complex structure [42].

Computer simulations using the molecular dynamics method [43] allowed the degree of polymer incorporation into the micelle structure to be analyzed. It was shown that the polymer links mainly enter the micelle polar shell. As a result, they additionally cover the surface of a hydrophobic domain and reduce the contact area with a polar solvent. The cited authors showed that the hydrophobic interaction is a major factor governing the aggregation of a neutral polymer with surfactant micelles.

\section{Molecular-Thermodynamic Model of Micellization and Formation of Surfactant-Polymer Complexes}

The aforementioned experimental results obtained while studying the interaction of neutral polymers with surfactants were generalized and described in the framework of the molecular thermodynamic theory of complex formation $[44,45]$. This theory is based on the theoretical description of the micellization thermodynamics [46] and extends it to the case of polymer-micellar complexes.

In the general form, the free energy of micellization includes five terms, with each of them corresponding to a separate physicochemical factor that influences

ISSN 2071-0194. Ukr. J. Phys. 2020. Vol. 65, No. 9 
the micellization process:

$$
\begin{aligned}
& \frac{\Delta \mu_{\mathrm{mic}}^{0}}{k T}=\frac{\Delta \mu_{\mathrm{hc} / \mathrm{w}}^{0}}{k T}-\ln \left(\frac{\Omega_{\mathrm{mic}}}{\Omega_{\mathrm{hc}}}\right)+\frac{\Delta \mu_{\sigma}^{0}}{k T}- \\
& -\ln \left(1-\frac{a_{p}}{a}\right)+\frac{\Delta \mu_{\mathrm{elec}}^{0}}{k T} .
\end{aligned}
$$

Here, the first term describes a change of the free energy associated with the variation of the local environment for the hydrophobic part of a surfactant molecule. The second term corresponds to the free energy change owing to a reduction of the conformational space for the hydrocarbon chain in the micelle, $\Omega_{\text {mic }}$, in comparison with that of the identical hydrocarbon chain in the liquid hydrocarbon phase, $\Omega_{\mathrm{hc}}$. The third term is associated with the formation of a phase interface between the micelle core and the solvent. The fourth term describes a contribution from the steric repulsion of the hydrophilic sections of surfactant molecules in the micelle shell (here, $a_{p} / a$ is the ratio between the area of the micelle surface occupied by the polar surfactant particles and the total micelle surface area). The forms of the first four terms in Eq. (3) and a detailed description of relevant parameters are given in work [47]. The last term in Eq. (3) corresponds to the electrostatic interaction between the charged parts of a surfactant molecule. It is described by an approximate solution of the nonlinear Poisson-Boltzmann equation [48].

The equilibrium concentration of micelles with the aggregation degree $N$ is determined by the formula

$X_{N}=X_{1}\left\{\exp \left(-\frac{\Delta \mu_{\mathrm{mic}}^{*}}{k T}\right)\right\}^{N}$,

where the parameter $\Delta \mu_{\text {mic }}^{*}$ is determined by minimizing the free energy [see formula (3)].

When being extended to the case of polymer association, the theoretical model of micellization, in its simplest variant, considers the effect of additional coating of the micelle hydrophobic core from the solvent medium by polymer links [45], which reduces the free energy of the system. A more detailed model of the process of polymer adsorption on the micelle surface was considered in work [44]. The cited authors applied a theoretical approach that was developed for the interaction of a polymer with colloidal particles [49]. They analyzed a model of the multimicelle complex formation, in which micelles are adsorbed step-by-step on a polymer chain, giving rise to the formation of the so-called pearl-necklace structure.

\section{Structural Analysis Using the SANS and Reflectometry Methods}

Considerable progress is observed in the study of the structure of micellar systems using the low-angle scattering of neutrons, as well as X-ray and synchrotron radiation $[50,51]$. The experimental dependence of the intensity of elastic coherent scattering on the scattering vector magnitude, $I(q)$, is interpreted in terms of the form factor $P(q)$ and the structure factor $S(q)$,

$I(q)=n_{p} P(q) S(q)$.

The form factor $P(q)$ is the square of the scattering amplitude $F(q)$ and describes the intensity of scattering on one micelle. It depends on the distribution of the scattering length density (SLD) inside the micelle, $\rho(\mathbf{r})$, or, more precisely, on the contrast value $\Delta \rho(\mathbf{r})-\rho_{0}$ with respect to the solvent $\mathrm{SLD} \rho_{0}$,

$P(q)=|F(q)|^{2}=\int_{\Omega}\left|\left(\rho(\mathbf{r})-\rho_{0}\right) e^{i \mathrm{kr}}\right|^{2} d \mathbf{r}$.

For micellar systems, model form factors - e.g., for a sphere, ellipsoid, cylinder, flexible cylinder, and so forth - are used as a rule [52].

The structure factor $S(q)$ takes the short-range order into account. It is a Fourier transform of the pair correlation function $g(r)$,

$S(q)=1+4 \pi n \int(g(r)-1) \frac{\sin (q r)}{q r} r^{2} d r$.

In practice, in order to determine the function $g(r)$, the Ornstein-Zernike equation should be solved in the mean-spherical approximation [53] with an interparticle interaction potential $V(r)$ described by the Coulombic potential of screened hard spheres with the effective diameter $\sigma$ :

$V(r)= \begin{cases}\pi \varepsilon_{o} \varepsilon \sigma^{2} \psi_{0}^{2} \frac{e^{-k(r-\sigma)}}{r}, & \text { if } r>\sigma, \\ \infty, & \text { if } r<\sigma,\end{cases}$

where $\psi$ is the surface potential associated with the micelle charge $z_{m}$ and the Debye radius $\kappa$,

$\psi_{0}=\frac{z_{m}}{\pi \varepsilon_{0} \varepsilon \sigma(2+\kappa \sigma)}$.

In the general case, the micelle shapes are nonspherical, and they are characterized by a certain size 

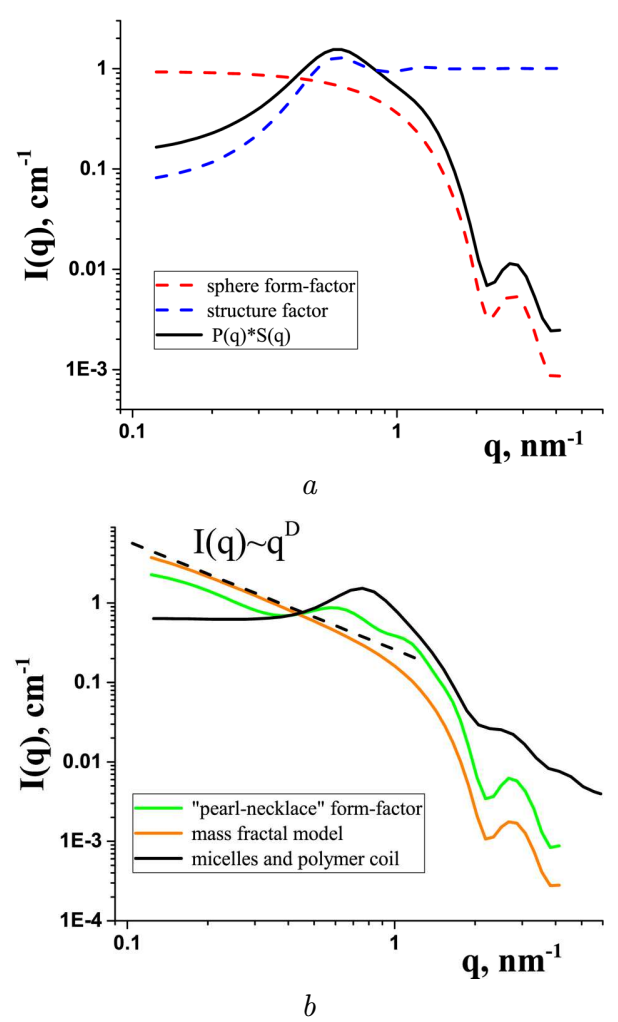

Fig. 4. Model scattering curves for micellar systems: the form factor of spheres $P(q)$ and the structure factor $S(q)(a)$. Model plots of form factors describing the scattering by surfactantpolymer complexes $(b)$. The mass fractal exponent $D$ varies from 1.5 to 2.5

distribution which is described by a polydispersity function. Therefore, two structure factors are calculated: (i) the effective structure factor $S^{\prime}(q)$, when considering all particles as spherical with the effective diameter $D_{\text {eff }}$, and (ii) the structure factor $S(q)$, when making allowance for the polydispersity and a deviation from the spherical micelle shape in the assumption that the micelle position does not depend on the micelle orientation and size [54],

$S(q)=1+\frac{|\langle F(q)\rangle|^{2}}{\left\langle|F(q)|^{2}\right\rangle}\left(S^{\prime}(q)-1\right)$,

where the notation $\langle\ldots\rangle$ means the averaging over the micelle size and orientation.

The approach described above, when being applied in a number of classical works, made it possible to obtain comprehensive information on the structure of micelles and their interaction [55-57]. Figures 4, a and $4, b$ exhibit model curves $I(q)$ that are used to describe experimental scattering data for micellar surfactant solution systems (Fig. 4, $a$ ) and for complexes formed with a polymer (Fig. $4, b$ ).

Experimental SANS data obtained for micellar systems of an ionic surfactant solution are described by a model curve corresponding to the scattering on charged spheres or ellipsoids, $I_{m}(q)$. For the systems with a neutral polymer and a surfactant concentration higher than the stoichiometric ratio, the scattering intensity is the sum of two contributions: the scattering by micelles, $I_{m}(q)$, and the scattering by the polymer ball structure, $I_{p}(q)$. At stoichiometric surfactant-to-polymer ratios, the scattering mainly occurs on complexes. In this case, it is described in the framework of the pearl-necklace (or mass-fractal) model [58].

The influence of the neutral PEG polymer on the structure and interaction of an anionic surfactant was studied using the SANS method within a wide concentration interval of substances with a varying molecular weight of the polymer $[29,59]$. The concentration dependences of the micelle aggregation number, the ionization degree, and the shape anisotropy parameter revealed tendencies to form spherical micelles in the structure of complexes with lower aggregation numbers and higher ionization degrees as compared with the case of free micelles. However, the characteristic distance between the micelles decreases at that as a result of their interaction with the polymer.

The neutron reflectometry method provides information on the structure of planar layered systems and phase interfaces. The experimentally measured quantity, the reflectance $R$, as a function of the normal component of the scattering vector magnitude $q$, $R(q)$, makes it possible to determine the distribution profile of the scattering length density into the depth of the researched system, $\rho(z)$ [60]. To study liquid systems, in particular, solutions of surfactants and polymers, the method of reflectometry with the horizontal orientation of the reflection surface is applied, which enables the free liquid/air interface (the liquid surface) to be analyzed [61]. Thus, the neutron reflectometry method is convenient for the structural analysis of the adsorbed surfactant layer or surfactantpolymer complexes at the liquid/air interface [62]. In work [63], the influence of an anionic surfactant on the profile of a polymer layer on a solid substrate 
was studied. Nowadays, along with the so-called classical reflectometry (specular reflection), the method of small-angle scattering at grazing incidence angles is also actively developed and used [64]. This method allows one to obtain the structural characteristics of nanoparticles located in the specimen surface plane.

\section{Conclusions}

Applied researches of multicomponent liquid systems including subsystems with self-organization properties are based on the results of studies of classical micellar systems. However, the synergistic nature of the interaction between the system components results in the appearance of phenomena that are not characteristic of separate subsystems. Therefore, it becomes necessary to apply approaches that were already developed when studying micellar systems and adapt them to multicomponent liquid systems, which was highlighted in this work by the example of micellar systems of aqueous surfactant solutions with the added polymer.

The challenging character of structural studies of liquid surfactant systems and surfactant-polymer systems in a wide range of applications is demonstrated. By an example of experimental works dealing with surfactants, surfactant-polymer liquid systems, and multicomponent systems with nanoparticles, the unique capabilities of the small-angle neutron scattering method are shown. Brief information about some modern complementary methods applied to perform the structural analysis of liquid systems with self-organization is reported.

1. B. Kronberg, K. Holmberg, B. Lindman. Surface Chemistry of Surfactants and Polymers (Wiley, 2014).

2. J. Kwak. Polymer-Surfactant Systems (CRC Press, 1998).

3. C. Tanford. Micelle shape and size. J. Phys. Chem. 76, 3020 (1972).

4. R. Zana, E.W. Kaler. Giant Micelles: Properties and Applications (CRC Press, 2007).

5. E.D. Goddard. Polymer-surfactant interaction: Part I. Uncharged water-soluble polymers and charged surfactants. In: Interactions of Surfactants with Polymers and Proteins (CRC Press, 2018), Chap. 4.

6. E.D. Goddard. Polymer-surfactant interaction: Part II Polymer and surfactant of opposite charge. Colloids Surf. 19, 301 (1986).

7. D. Langevin. Complexation of oppositely charged polyelectrolytes and surfactants in aqueous solutions. A review. Adv. Colloid Interface Sci. 147-148, 170 (2009).
8. B. Cattoz, W.M. De Vos, T. Cosgrove et al. Manipulating interfacial polymer structures through mixed surfactant adsorption and complexation. Langmuir 28, 6282 (2012).

9. S. Mehan, A.J. Chinchalikar, S. Kumar et al. Small-angle neutron scattering study of structure and interaction of nanoparticle, protein, and surfactant complexes. Langmuir 29, 11290 (2013).

10. P. Hansson, B. Lindman. Small-angle neutron scattering study of structure and interaction of nanoparticle, protein, and surfactant complexes. Curr. Opin. Colloid Interface Sci. 1, 604 (1996).

11. K.C. Tam, E. Wyn-Jones. Insights on polymer surfactant complex structures during the binding of surfactants to polymers as measured by equilibrium and structural techniques. Chem. Soc. Rev. 35, 693 (2006).

12. J.N. Phillips. The energetics of micelle formation. Trans. Faraday Soc. 51, 561 (1955).

13. JC.G. Bell, C.J.W. Breward, P.D. Howell et al. Macroscopic modeling of the surface tension of polymer-surfactant systems. Langmuir 23, 6042 (2007).

14. J. Goronja, A. Janosevic-Lezaic, B. Dimitrijevic et al. Determination of critical micelle concentration of cetyltrimethylammonium bromide: Different procedures for analysis of experimental data. Hem. Industr. 70, 485 (2016).

15. V.J. Sovilj, L.B. Petrovic. Influence of molecular characteristics of nonionic cellulose ethers on their interaction with ionic surfactant investigated by conductometry. Colloid Polym. Sci. 284, 334 (2005).

16. F.M. Winnik, S.T.A. Regismond. Fluorescence methods in the study of the interactions of surfactants with polymers. Colloids Surf. A 118, 1 (1996).

17. D. Bica, L. Vékás, M.V. Avdeev et al. Sterically stabilized water based magnetic fluids: Synthesis, structure and properties. J. Magn. Magn. Mater. 311, 17 (2007).

18. E. Tombacz, D. Bica, A. Hajdu et al. Surfactant double layer stabilized magnetic nanofluids for biomedical application. J. Phys. Condens. Matter 20, 204103 (2008).

19. A.V. Nagornyi, V. Socoliuc, V.I. Petrenko et al. Structural characterization of concentrated aqueous ferrofluids. J. Magn. Magn. Mater. 501, 166445 (2020).

20. C. Alexiou, R. Schmid, R. Jurgons et al. Targeted tumor therapy with "magnetic drug targeting": Therapeutic efficacy of ferrofluid bound mitoxantrone. In: Ferrofluids. Magnetically Controllable Fluids and Their Applications. Edited by S. Odenbach (Springer, 2002), p. 233.

21. B. Gleich, J. Weizenecker. Tomographic imaging using the nonlinear response of magnetic particles. Nature 435, 1214 (2005).

22. E. Illes, M. Szekeres, E. Kupcsik et al. PEGylation of surfacted magnetite core-shell nanoparticles for biomedical application. Colloids Surf. A 460, 429 (2014).

23. V. Zavisova, M. Koneracka, M. Muckova et al. Magnetic fluid poly(ethylene glycol) with moderate anticancer activity. J. Magn. Magn. Mater. 323, 1408 (2011).

24. V.I. Petrenko, O.P. Artykulnyi, L.A. Bulavin et al. On the impact of surfactant type on the structure of aqueous ferrofluids. Colloids Surf. A 541, 222 (2018). 
25. M.V. Avdeev, A.V. Feoktystov, P. Kopcansky et al. Structure of water-based ferrofluids with sodium oleate and polyethylene glycol stabilization by small-angle neutron scattering: Contrast-variation experiments. J. Appl. Crystallogr. 43, 959 (2010).

26. M. Kubovcikova, I.V. Gapon, V. Zavisova et al. On the adsorption properties of magnetic fluids: Impact of bulk structure. J. Magn. Magn. Mater. 427, 67 (2017).

27. M.V. Avdeev, V.I. Petrenko, I.V. Gapon et al. Comparative structure analysis of magnetic fluids at interface with silicon by neutron reflectometry. Appl. Surf. Sci. 352, 49 (2015).

28. V.I. Petrenko, M.V. Avdeev, V.M. Garamus et al. Micelle formation in aqueous solutions of dodecylbenzene sulfonic acid studied by small-angle neutron scattering. Colloids Surf. A 369, 160 (2010).

29. O.P. Artykulnyi, V.I. Petrenko, L.A. Bulavin et al. Effect of polyethylene glycol on the structure of micellar solutions of sodium oleate in water according to the data of smallangle neutron scattering. J. Surf. Invest. Synchr. Neutr. Techn. 12, 1142 (2018).

30. V.I. Petrenko, M.V. Avdeev, L.A. Bulavin et al. Effect of surfactant excess on the stability of low-polarity ferrofluids probed by small-angle neutron scattering. Crystallogr. Rep. 61, 121 (2016).

31. M.V. Avdeev, D. Bica, L. Vekas et al. Comparative structure analysis of non-polar organic ferrofluids stabilized by saturated mono-carboxylic acids. J. Colloid Interface Sci. 334, 37 (2009).

32. V.I. Petrenko, M.V. Avdeev, V.L. Aksenov et al. Effect of surfactant excess in non-polar ferrofluids probed by smallangle neutron scattering. Solid State Phenom. 152-153, 198 (2009).

33. V.I. Petrenko, V.L. Aksenov, M. V. Avdeev et al. Analysis of the structure of aqueous ferrofluids by the smallangle neutron scattering method. Phys. Solid State. 52, 974 (2010).

34. M.D. Chavanpatil, A. Khdair, J. Panyam et al. Surfactantpolymer nanoparticles: A novel platform for sustained and enhanced cellular delivery of water-soluble molecules. Pharm. Res. 24, 803 (2007).

35. S. Qi, S. Roser, K.J. Edler et al. Insights into the role of polymer-surfactant complexes in drug solubilisation/stabilisation during drug release from solid dispersions. Pharm. Res. 30, 290 (2013).

36. B. Nyström, K. Thuresson, B. Lindman et al. Rheological and dynamic light-scattering studies on aqueous solutions of a hydrophobically modified nonionic cellulose ether and its unmodified analogue. Langmuir 11, 1994 (1995).

37. B. Nyström, B. Lindman. Dynamic and viscoelastic properties during the thermal gelation process of a nonionic cellulose ether dissolved in water in the presence of ionic surfactants. Macromolecules 28, 967 (1995).

38. P. Hansson, B. Lindman. Surfactant-polymer interactions. Curr. Opin. Colloid Interface Sci 1, 604 (1996).
39. R. Zana, P. Lianos, J. Lang et al. Fluorescence probe studies of the interactions between poly(oxyethylene) and surfactant micelles and microemulsion droplets in aqueous solutions. J. Phys. Chem. 89, 41 (1985).

40. G. Bastiat, B. Grassl, J. Francois. Micellar copolymerization of associative polymers: Study of the effect of acrylamide on sodium dodecyl sulfate-poly(propylene oxide) methacrylate mixed micelles. J. Colloid Interface Sci. 289, 359 (2005).

41. R. Meszaros, I. Varga, T. Gilanyi. Effect of polymer molecular weight on the polymer/surfactant interaction J. Phys. Chem. B 109, 13538 (2005).

42. J. Van Stam, N. Wittouck, F.C. De Schryver. The role of polymer flexibility on the interaction with surfactant micelles: poly(vinyl alcohol) and sodium dodecyl sulphate aqueous micelle interactions studied by dynamic fluorescence quenching Can. J. Chem. 73, 1765 (1995).

43. B.Z. Shang, Z. Wang, R.G Larson. Molecular dynamics simulation of interactions between a sodium dodecyl sulfate micelle and a poly(ethylene oxide) polymer. J. Phys. Chem. B 112, 2888 (2008).

44. Y.J. Nikas, D. Blankschtein. Complexation of nonionic polymers and surfactants in dilute aqueous solutions. Langmuir. 10, 3512 (1994).

45. R. Nagarajan. Thermodynamics of nonionic polymermicelle association. Colloids Surf. 13, 1 (1985).

46. S. Puvvada, D. Blankschtein. Molecular-thermodynamic approach to predict micellization, phase behavior and phase separation of micellar solutions. I. Application to nonionic surfactants. J. Chem. Phys. 92, 3710 (1990).

47. A. Naor, S. Puvvada, D. Blankschtein. An analytical expression for the free energy of micellization. J. Phys. Chem. 96, 7830 (1992).

48. H. Ohshima, T.W. Healy, L.R. White. Accurate analytic expressions for the surface charge density/surface potential relationship and double-layer potential distribution for a spherical colloidal particle. J. Colloid Interface Sci. 90, 17 (1982).

49. E. Eisenriegler, A. Hanke, S. Dietrich. Polymers interacting with spherical and rodlike particles. Phys. Rev. E 54, 1134 (1996).

50. L.A. Feigin D.I. Svergun, Structure Analysis by SmallAngle X-Ray and Neutron Scattering (Springer, 1987) [ISBN: 978-1-4757-6626-4].

51. L.A. Bulavin. Neutron Diagnostics of Matter Liquid State (Chornobyl, 2012) (in Ukrainian) [ISBN: 978-966-026196-9].

52. J.S. Pedersen. Analysis of small-angle scattering data from colloids and polymer solutions: Modeling and least-squares fitting. Adv. Colloid Interface Sci. 70, 171 (1997).

53. J.P. Hansen, J.B. Hayter. A rescaled MSA structure factor for dilute charged colloidal dispersions. Mol. Phys. 46, 651 (1982).

54. M. Kotlarchyk, S. Chen. Analysis of small angle neutron scattering spectra from polydisperse interacting colloids. J. Chem. Phys. 79, 2461 (1983).

ISSN 2071-0194. Ukr. J. Phys. 2020. Vol. 65, No. 9 
55. L.A. Bulavin, V.M. Garamus, T.V. Karmazina et al. Measurements of structural and electrostatic parameters and surface tension of micelles of an ionic surfactant versus concentration, ionic strength of solution and temperature by small-angle neutron scattering. Colloids Surf. A 131, 137 (1998).

56. M. Kakitani, T. Imae, M. Furusaka. Investigation of mixed micelles of dodecyldimethylamine oxide and sodium dodecyl sulfate by SANS: Shape, size, charge, and interaction. J. Phys. Chem. 99, 16018 (1995).

57. W.R. Chen, P.D. Butler, L.J. Magid. Incorporating intermicellar interactions in the fitting of SANS data from cationic wormlike micelles. Langmuir 22, 6539 (2006).

58. O.P. Artykulnyi, A.V. Shibaev, M.M. Avdeev et al. Structural investigations of poly(ethylene glycol)-dodecylbenzenesulfonic acid complexes in aqueous solutions. J. Mol. Liq. 308, 113045 (2020).

59. O.P. Artykulnyi, V.I. Petrenko, L.A. Bulavin et al. Impact of poly (ethylene glycol) on the structure and interaction parameters of aqueous micellar solutions of anionic surfactants. J. Mol. Liq. 276, 806 (2019).

60. X.L. Zhou, S.H. Chen. Theoretical foundation of X-ray and neutron reflectometry. Phys. Rep. 257, 223 (1995).

61. M.V. Avdeev, V.I. Bodnarchuk, V.I. Petrenko et al. Neutron time-of-flight reflectometer GRAINS with horizontal sample plane at the IBR-2 reactor: Possibilities and prospects. Cryst. Rep. 62, 1002 (2017).

62. D.J.F. Taylor, R.K. Thomas, J. Penfold. Polymer/surfactant interactions at the air/water interface. Adv. Colloid Interface Sci. 132, 69 (2007).

63. M. Moglianetti, J.R.P. Webster, S. Edmondson et al. A neutron reflectivity study of surfactant self-assembly in weak polyelectrolyte brushes at the sapphire-water interface. Langmuir 27, 4489 (2011).

64. S. Jaksch, T. Gutberlet, P. Müller-Buschbaum. Grazingincidence scattering-status and perspectives in soft matter and biophysics. Curr. Opin. Colloid Interface Sci. 42, 73 (2019).

Received 10.04.20.

Translated from Ukrainian by O.I. Voitenko

О.П. Артикулъний, В.І. Петренко,

М.В.Авдєєв, Л.А.Булавін

КОМПЛЕКСИ МІЦЕЛ

ПОВЕРХНЕВО-АКТИВНИХ РЕЧОВИН

З ПОЛІМЕРАМИ У ВОДНИХ

РІДИННИХ СИСТЕМАХ

$\mathrm{P}$ е $з$ ю м е

Огляд присвячений структурним дослідженням самоорганізації та агрегації у водних рідинних системах поверхневоактивних речовин (ПАР) та полімерів. У роботі здебільшого наведено результати досліджень взаємодії іонних ПАР з неіонними полімерами. Розглянуто можливості методу малокутового розсіяння нейтронів, а також рефлектометрії, для дослідження структури міцел, агрегатів міцел з полімерами та складних багатокомпонентних систем з наночастинками, де важливу роль відіграють процеси міцелоутворення та комплексоутворення. Наведено коротку інформацію про деякі комплементарні методи структурного аналізу рідинних систем із самоорганізацією. 